\title{
RARE PRESENTATION OF HYPERREACTIO LUTEINALIS IN PREGNANCY: A CASE REPORT
}

\author{
Arpana Verma1, Nagarathna Kuntoji2
}

1 Post Graduate, Department of Obstetrics and Gynaecology, Shri Nijalingappa Medical College, Bagalkot.

2 Professor and Unit Chief, Department of Obstetrics and Gynaecology, Shri Nijalingappa Medical College, Bagalkot.

\begin{abstract}
Hyperreactio luteinalis is a benign condition associated with bilateral and in rare cases unilateral ovarian enlargement due to theca lutein cysts. Hyperreactio luteinalis is usually found incidentally at caesarean section, which can appear anaplastic and lead to unnecessary ovarian resection. It is estimated that approximately $60 \%$ of the cases of hyperreactio luteinalis unassociated with trophoblastic disease occur with normal singleton pregnancy. There are multiple benign ovarian lesions including hyperreactio luteinalis that can mimic ovarian neoplasms. Accordingly, it is important to exclude these from the differential diagnosis via a wedge biopsy and frozen section to avoid unnecessary surgical excision. A 28-year-old woman, G2P1L0, presented at 36 weeks and 2 days of gestation with a singleton pregnancy, with previous LSCS, with mild preeclampsia with left multicystic ovarian enlargement with subsequent virilising features, and a normal beta hCG. The patient underwent a low transverse caesarean section. The left ovary had an anaplastic appearance for which left salpingo-oophorectomy was done. Histopathological examination revealed multiple benign theca lutein cysts.
\end{abstract}

\section{KEYWORDS}

Hyperreactio Luteinalis, Caesarean Section, Theca Lutein Cyst, Ovarian Hyperstimulation.

HOW TO CITE THIS ARTICLE: Verma A, Kuntoji N. Rare presentation of hyperreactio luteinalis in pregnancy: a case report. J. Evolution Med. Dent. Sci. 2016;5(52):3471-3473, DOI: 10.14260/jemds/2016/801

\section{INTRODUCTION}

Hyperreactio Luteinalis (HL) is a rare entity caused by presence of numerous luteinized follicle cysts resulting in variable degrees of ovarian enlargement.[1] The aetiology of this condition is unknown, but it is believed to be caused by either prolonged or excessive stimulation of the ovaries by gonadotropins or increased ovarian sensitivity to gonadotropins.[2] It is known to be typically associated with gestational trophoblastic disease and ovarian stimulation for ovulation. Association with hyperemesis gravidarum, multiple pregnancy, and foetal hydrops has also been reported. However, it is very rare in a spontaneously conceived normal singleton pregnancy.[1,2] HL sometimes is revealed accidentally at the time of routine pelvic ultrasound examination because patients with HL may be asymptomatic. Unnecessary laparotomy during pregnancy can be performed by physicians because of its rarity and unfamiliarity. Since Burger. ${ }^{3}$ described the first case of HL unassociated with trophoblastic disease in 1938, about 80 reports have been published. Still, only sporadic cases of HL, especially conditions managed conservatively, have been reported.

\section{CASE REPORT}

A 28-year-old G2P1LO came with history of amenorrhoea of eight and a half month and complain of headache since 1 day. On examination, patient had virilisation. Uterus was 32 weeks size, relaxed with cephalic presentation. Foetal heart rate was $140 /$ min and regular.

Financial or Other Competing Interest: None.

Submission 12-05-2016, Peer Review 07-06-2016.

Acceptance 13-06-2016, Published 30-06-2016.

Corresponding Author:

Arpana Verma,

Room No. 43, PG Hostel,

S.N. Medical College,

Hanagal Shri Kumareshwar Hospital and Research Centre,

Bagalkot, Karnataka.

E-mail: dr.arpanaverma@gmail.com

DOI: $10.14260 /$ jemds $/ 2016 / 801$
There was separate mass felt in left side extending from iliac region to hypochondrium, soft to cystic in consistency, freely mobile only in horizontal direction and was non-tender. On per vaginal examination, cervix was soft, posterior os was closed. On bimanual examination, cystic mass felt in the left fornix. Patient underwent elective caesarean section at 37 weeks with outcome being live male baby of $2.7 \mathrm{~kg}$. Intraoperatively, there was left-sided large multiloculated cyst of approximately $20 * 20 \mathrm{~cm}$ with thick mucinous content for which left salpingo-oophorectomy was done. Right tube and ovary were absent. May be, patient underwent right salpingooophorectomy during previous caesarean section 3 years back. Patient gives history of mass per abdomen during previous pregnancy for which records not available. Postoperative period was uneventful and patient was discharged on day 10.

\section{Investigation}

Ultrasonography showed single live intrauterine foetus of 35 weeks with good cardiac activity and left adnexal large multicystic mass with multiple septations mass extending from under surface of spleen to left iliac fossa.

\section{Intraoperative Findings}

Left side cyst was present measuring approximately $20 * 20 \mathrm{~cm}$, multiloculated, one locule accidently got ruptured. Thick milky fluid was drained. The left ovary was not separately visualised. The right tube and ovary were absent (? Salpingooophorectomy done during previous LSCS). 


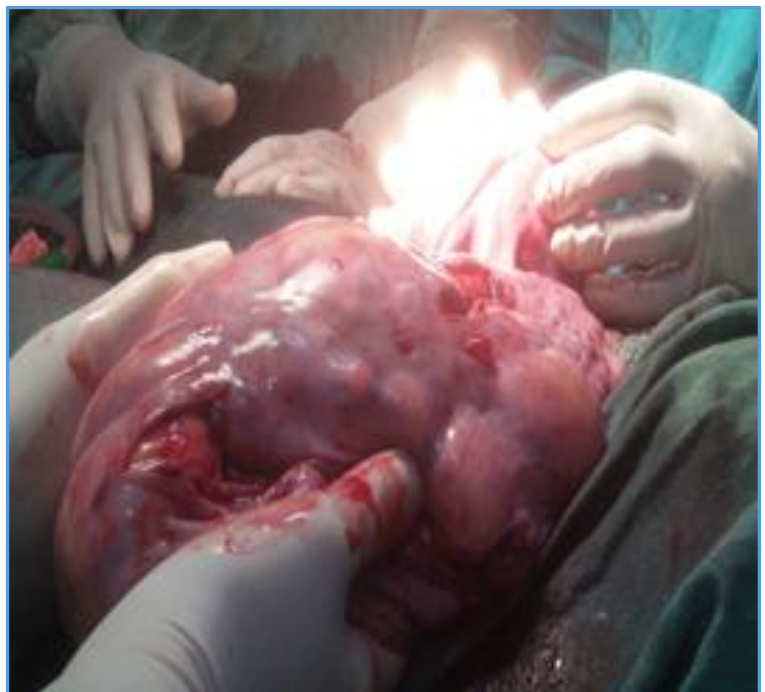

Fig. 1: Intraoperative Finding showing Large Left Multiloculated Adnexal Mass

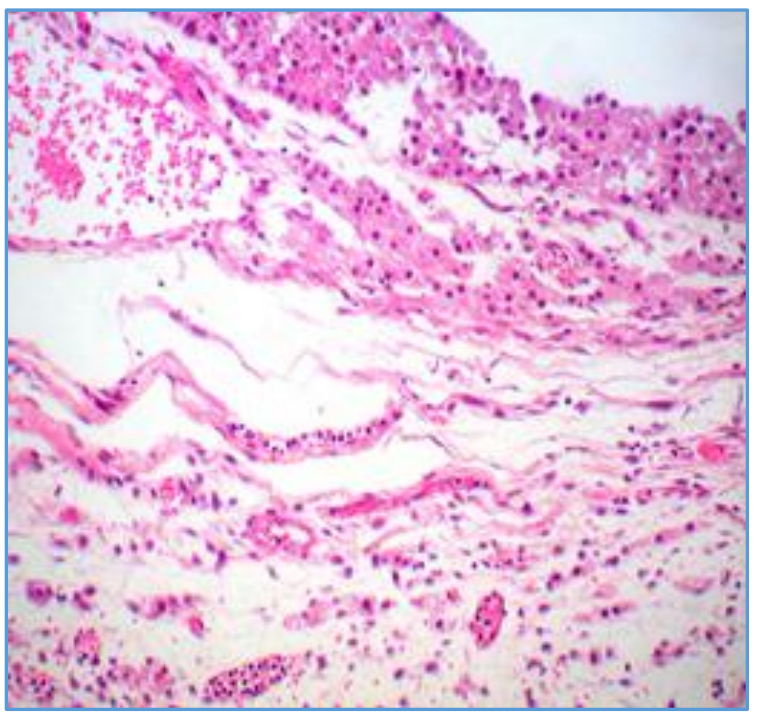

Fig. 2: Microscopically seen Clusters of Luteinized Cells in Oedematous Stroma

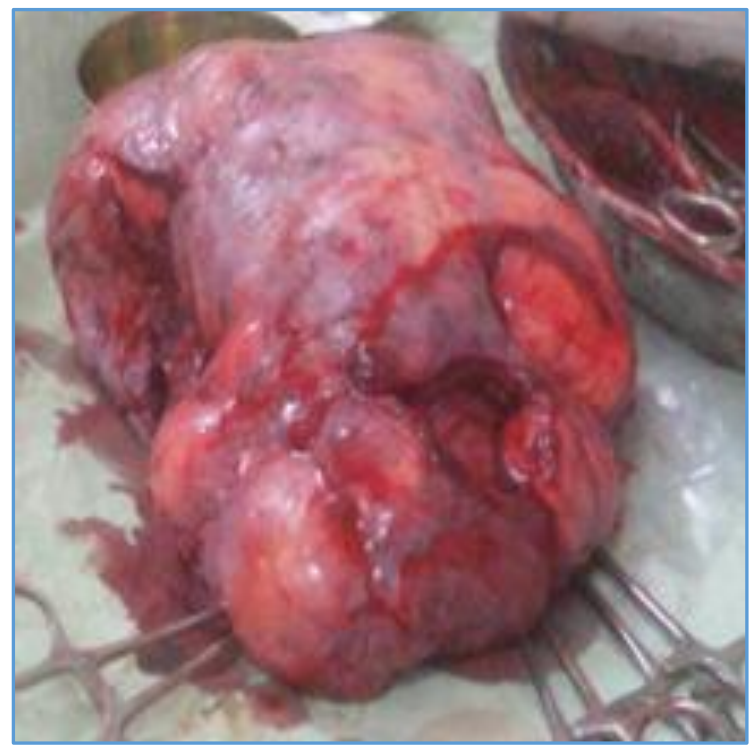

Fig. 3: Macroscopic Appearance of the Cystic Ovary

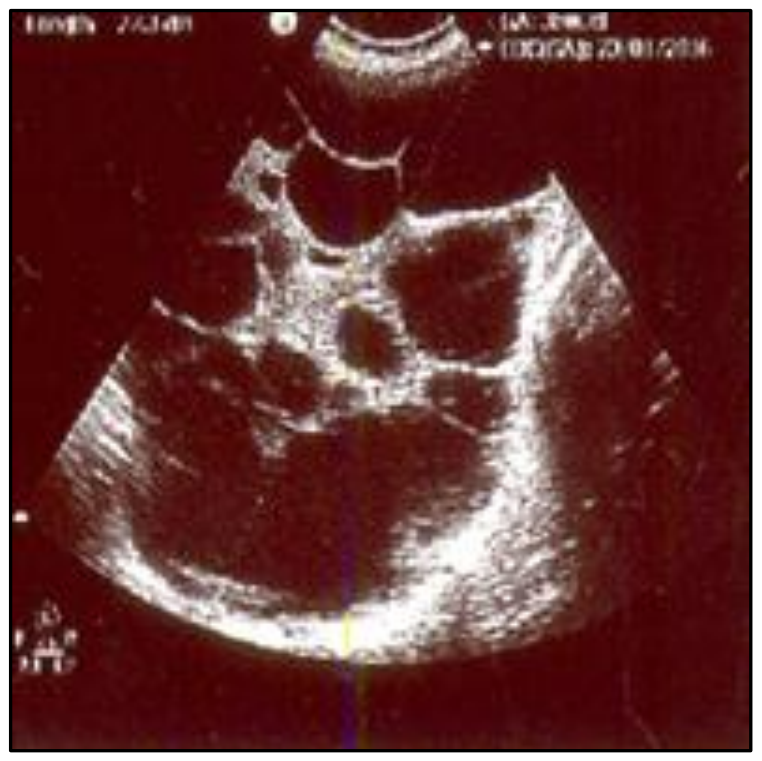

Fig. 4: USG showing Large Left Multiloculated Adnexal Mass

\section{DISCUSSION}

Hyperreactio luteinalis is a rare entity in which enlarged cystic ovaries develop during spontaneously conceived pregnancy. The reaction is sometimes associated with elevated serum hCG levels and sometimes normal serum levels and is characterized by an exaggerated ovarian response to this hormone. The ovaries become markedly enlarged with multiple luteinized follicular cysts. Histologically, cysts are filled with clear to blood-tinged fluid or clotted blood with a smooth lining composed of an inner layer of granulose cells and an outer layer of markedly luteinized and hypertrophic theca interna cells. The ovarian stroma is typically oedematous. ${ }^{[3,4]}$ The presentation of bilateral enlarged multicystic ovaries can also be seen in ovarian hyperstimulation syndrome, although these differ in that the ovaries in ovarian hyperstimulation syndrome undergo rapid expansion accompanied by systemic fluid shifts following pharmacologic stimulation of follicular development and ovulation. ${ }^{[5,6]}$

Hyperreactio luteinalis has been observed with multiple gestations, gestational trophoblastic disease, malignancy, renal dysfunction, conditions that may be associated with elevated hCG levels, and abnormal or enlarged placenta. $[4,7]$

It has been previously reported that symptoms, such as maternal virilisation, occur in approximately $15 \%$ of women with HL. Haemoperitoneum and ovarian torsion are less common complications.[3,8] It has been suggested that HL occurs more commonly in primigravida and more commonly in younger patients. On ultrasound examination, hyperreactio luteinalis is characterized by large adnexal masses that consist of many thin-walled small theca lutein cysts giving it the appearance of a 'spoke wheel'.

Most of these patients are asymptomatic. This condition, being self-limiting in course, most often does not need any intervention/medications. ${ }^{[9]}$ Based on the size of these multicystic ovaries, the patients may have pain or may undergo torsion, cyst rupture, or pelvic entrapment of enlarged ovary, which then becomes a surgical emergency.[10] These patients may develop hyperandrogenism due to elevated $\beta$ hCG levels and require appropriate management. 


\section{REFERENCES}

1. Caspi E, Schreyer P, Bukovsky J. Ovarian lutein cysts in pregnancy. Obstet Gynaecol 1973;42(3):388-98.

2. Abu-Louz SK, Ahmed AA, Swan RW. Spontaneous ovarian hyperstimulation syndrome with pregnancy. Am J Obstet Gynaecol 1997;177(2):476-7.

3. Wajda KJ, Lucas JG, Marsh WL. Hyperreactio luteinalis: benign disorder masquerading as an ovarian neoplasm. Arch Pathol Lab Med 1989;113(8):921-5.

4. Cunningham FG, Leveno KJ, Bloom SL, et al. Maternal physiology. In: Obstetrics W 23 $3^{\text {rd }}$ ed. New York, NY: McGraw-Hill Medical 2009:107-35.

5. Haimov-Kochman R, Yanai N, Yagel S, et al. Spontaneous ovarian hyperstimulation syndrome and hyperreactio luteinalis are entities in continuum. Ultrasound Obstet Gynaecol 2004;24(6):675-8.

6. Foulk RA, Martin MC, Jerkins GL, et al. Hyperreactio luteinalis differentiated from severe ovarian hyperstimulation syndrome in a spontaneously conceived pregnancy. Am J Obstet Gynaecol 1997;176(6):1300-2.
7. Al-Ramahi M, Leader A. Case report: hyperreactio luteinalis associated with chronic renal failure. Hum Reprod 1999;14(2):416-8.

8. Schnorr JA, Miller H, Davis JR, et al. Hyperreactio luteinalis associated with pregnancy: a case report and review of the literature. Am J Perinatol 1996;13(2):95-7.

9. Cho AR, Song TB, Kim HJ, et al. Vaginal delivery in a spontaneously conceived singleton pregnancy complicated with hyperreactio luteinalis: a case report. J Womens Med 2011;4(2):53-6.

10. Lynn KN, Steinkeler JA, Wilkins-Haug LE, et al. Hyperreactio luteinalis (enlarged ovaries) during the second and third trimesters of pregnancy: common clinical associations. J Ultrasound Med 2013;32(7): 1285-9. 